Tascón H., D. C., Cabra C., M. Y., y Lima R., W. E. (2021). Propuesta para la sistematización y evaluación de experiencias de Responsabilidad Social Universitaria, una ruta para su mejora, visibilidad y réplica. Plumilla Educativa, 28 (2), 81-106. DOI: 10.30554/pe.2.4313.2021.

\title{
Propuesta para la sistematización y evaluación de experiencias de Responsabilidad Social Universitaria, una ruta para su mejora, visibilidad y réplica
}

\author{
Diana Carolina Tascón Hoyos ${ }^{1}$ \\ María Yolanda Cabra Copete ${ }^{2}$ \\ Wilma Edith Lima Ravelo ${ }^{3}$
}

\begin{abstract}
Resumen
El surgimiento de nuevos desafíos para la educación superior con respecto de la Responsabilidad Social Universitaria (RSU), ha puesto de manifiesto la necesidad de desarrollar estrategias que permitan gestionar y dar a conocer de manera efectiva los esfuerzos que la academia realiza para su entorno. En este artículo se propone una ruta para la sistematización y evaluación de experiencias de RSU cuya
\end{abstract}

1 Diana Carolina Tascón Hoyos. Magister en Ingeniería Industrial, Universidad Distrital Francisco José de Caldas. Docente investigador de la Universidad Distrital Francisco José de Caldas (Bogotá). ORCID: https://orcid.org/0000-0002-8935-3800; Correo electrónico: dctasconh@udistrital.edu.co

2 María Yolanda Cabra Copete. Magister en Educación, Universidad Santo Tomás. Docente investigador de la Universidad Santo Tomás (Bogotá). ORCID: https://orcid.org/0000-00034254-8851; Correo electrónico: mariacabra@ustadistancia.edu.co

3 Wilma Edith Lima Ravelo. Magister en Gestión de Empresas Cooperativas y del sector Solidario, Universidad de Mondragón. Docente investigador de la Universidad Santo Tomás (Bogotá). ORCID: https://orcid.org/0000-0002-3568-3230; Correo electrónico: wilmalima@ustadistancia.edu.co 
aplicación permitiría mejorar su gestión; pues, entre otros beneficios, la sistematización y la aplicación de modelos de evaluación permiten visibilizar resultados ante actores sociales involucrados, así como organizar y gestionar los aprendizajes. La Universidad Santo Tomás de Colombia se tomó como referente de contextualización.

Palabras clave: Responsabilidad Social Universitaria, Sistematización de experiencias, Educación Superior, evaluación de experiencias, visibilidad.

\section{Proposal for the systematization and} evaluation of University Social Responsibility experiences, a route for its improvement, visibility and replication

\section{Abstract}

The emergence of new challenges for higher education in relation to University Social Responsibility (USR) has highlighted the need to develop strategies that effectively manage and publicize the efforts that the academy makes for its environment. This article proposes a route for the systematization and evaluation of USR experiences, its application would improve RSU management, since within other benefits, the systematization and evaluation models allow visibility of results among the social actors involved and favors the organization and management of learning. The Universidad Santo Tomás in Colombia was taken as a contextualization reference.

Key words: University Social Responsibility, Systematization of experiences, Higher Education, evaluation of experiences, visibility.

Proposta de sistematização e avaliação de experiências de Responsabilidade Social Universitária, caminho para melhoria, visibilidade e replicação

\section{Resumo}

O surgimento de novos desafios para o ensino superior em relação à Responsabilidade Social da Universidade (USW), destacou a 
necessidade de desenvolver estratégias que gerenciem e divulguem efetivamente os esforços que a academia faz para seu ambiente. Este artigo propõe um caminho para a sistematização e avaliação das experiências de RSU, cuja aplicação melhoraria sua gestão; Assim, entre outros benefícios, a sistematização e aplicação de modelos de avaliação permitem tornar os resultados visíveis aos atores sociais envolvidos, bem como organizar e gerenciar o aprendizado. A Universidade Santo Tomás da Colômbia foi tomada como referência para contextualização.

Palavras-chave: Responsabilidade Social Universitária, Sistematização de experiências, Ensino Superior, avaliação de experiências, visibilidade.

\section{Introducción 4}

La evolución de la Responsabilidad Social Universitaria (en adelante RSU), ha traído consigo un nuevo paradigma para la Universidad, pasando de la labor asistencialista en la sociedad al desarrollo de proyectos y actividades que promueven el empoderamiento de las comunidades y el crecimiento social y empresarial. El trabajo realizado por la Universidad con comunidades, con el sector productivo y con el Estado, cuyo objetivo es integrar la academia con la sociedad propendiendo por la solución de problemáticas que a su vez aporten a la evolución y pertinencia del currículo, en muchas ocasiones permanece invisible para buena parte del medio académico y social. Esta situación puede dar lugar a duplicidad de proyectos que abordan una misma temática, actividades desconectadas, comunidades sobre-diagnosticadas, así como deficiencias en la propagación de los saberes dentro de la comunidad académica y la sociedad que puede beneficiarse de las experiencias y aprendizajes logrados.

Por lo anterior, el artículo tiene como objetivo, a partir de una revisión de la literatura, diseñar una ruta de sistematización de experiencias que permita su reflexión, difusión y mejora, fortaleciendo los vínculos entre los diferentes actores e interesados en las estrategias de RSU que desarrollan

\footnotetext{
${ }^{4}$ Este artículo es derivado del proyecto de investigación: “Caracterización de experiencias significativas de la Proyección Social de los Centros de Atención Universitaria (CAU) pertenecientes a la Vicerrectoría Universidad Abierta y a Distancia (VUAD), Universidad Santo Tomás, durante el periodo 2012 a 2016", desarrollado desde el año 2017
} 
las Universidades. Esta ruta tendrá el potencial de usarse posteriormente para desarrollar trabajos colaborativos de socialización y evaluación de experiencias de RSU, para identificar territorios no explorados, necesidades no cubiertas, así como trabajos que puedan integrarse, complementarse o que estén resultando redundantes.

Así, se parte de la presentación de la metodología empleada, donde se incluyen una revisión de literatura, el estudio de estrategias para la sistematización de experiencias, una revisión de modelos de evaluación de RSU, para finalmente, y tomando lo anterior como insumo, cumplir con el objetivo fijado: diseñar una ruta de sistematización y evaluación de experiencias de RSU.

A fin de contar con una situación en la que contextualizar lo planteado, se aborda como caso de referencia lo trabajado en el marco de la RSU en la Universidad Santo Tomás de Colombia, en adelante USTA. Al revisar las experiencias de RSU en esta institución, esto en términos de su registro y valoración, se encontró que las acciones desarrolladas requieren de una estrategia sistemática y estandarizada que permita visibilizar su impacto y mejorar su registro y favorecer su gestión, lo que no dista de la situación descrita por López Vélez, (2016), quien señala la falta de un sistema para el registro sistemático de datos y documentación de experiencias de RSU que permita analizar lo que se ha hecho, evaluarlo y mejorarlo. La ruta planteada busca satisfacer estas necesidades y brindar un marco referencial para otras instituciones.

\section{Metodología}

Buscando responder al objetivo del proceso -sistematizar las experiencias de RSU visibilizando su impacto, favoreciendo la réplica y mejora continuase realizó un proceso mediante un trabajo de orden cualitativo, considerando que las experiencias "son procesos vitales y únicos (...), inéditos e irrepetibles" (Jara, 2011). Se acude a la sistematización de experiencias como enfoque de investigación que permite el desarrollo del proceso, porque ésta produce conocimientos y aprendizajes y permite comprender las experiencias teóricamente (Jara, 2011), lo cual va en línea con el propósito de interpretar y reconstruir lo vivido desde una perspectiva crítica. De esta forma, las experiencias realizadas en el marco de la Proyección Social pueden adquirir nuevos sentidos y aportar a la 
construcción de conocimiento en la Universidad. La metodología empleada, como se mencionó, incluye varias actividades enmarcadas dentro de la sistematización, que fueron aportando al desarrollo de la propuesta de la Ruta. La Tabla 1 y la Figura 1 sintetizan el proceso de construcción de la propuesta, el cual, en sí mismo, constituyó una experiencia, facilitando la inmersión del grupo en las actividades de sistematización.

\section{Tabla 1. Metodología empleada.}

\begin{tabular}{|c|c|c|}
\hline Actividad & Descripción & Producto \\
\hline $\begin{array}{l}\text { Revisión de la } \\
\text { literatura }\end{array}$ & $\begin{array}{l}\text { Revisión de la literatura sobre: } \\
\text { Responsabilidad Social, } \\
\text { Responsabilidad Social Empresarial, } \\
\text { Responsabilidad Social Universitaria y } \\
\text { Responsabilidad Social Universitaria } \\
\text { en la Universidad caso de estudio, } \\
\text { sistematización de experiencias y su } \\
\text { evaluación }\end{array}$ & $\begin{array}{l}\text { Definición de referentes } \\
\text { bibliográficos para el diseño } \\
\text { de la ruta }\end{array}$ \\
\hline $\begin{array}{l}\text { Diseño de ruta } \\
\text { para la } \\
\text { sistematización } \\
\text { y evaluación } \\
\text { de } \\
\text { experiencias }\end{array}$ & $\begin{array}{l}\text { Estudiar estrategias existentes para la } \\
\text { sistematización de experiencias } \\
\text { Analizar modelos existentes que } \\
\text { permitan la evaluación de las } \\
\text { experiencias } \\
\text { Tomando en cuenta lo encontrado en } \\
\text { el desarrollo de las actividades } \\
\text { previas y las necesidades a las que } \\
\text { pretende atender las ruta, ésta fue } \\
\text { diseñada }\end{array}$ & $\begin{array}{l}\text { Selección de una estrategia } \\
\text { de sistematización de } \\
\text { experiencias a usar en la } \\
\text { ruta propuesta } \\
\text { Selección de un modelo de } \\
\text { evaluación de experiencias } \\
\text { de RSU a usar en la ruta } \\
\text { propuesta } \\
\text { Ruta para la sistematización } \\
\text { y evaluación de experiencias }\end{array}$ \\
\hline
\end{tabular}

Fuente: Elaboración propia.

\section{Responsabilidad Social}

El término Responsabilidad Social ha sido abordado desde diferentes perspectivas: académica, social y empresarial, entre otras. Los líderes de opinión, pensadores, los organismos internacionales y las corporaciones han realizado sus aportes frente al tema, buscando responder a los retos que plantea el mundo ante situaciones como el deterioro ambiental, la injusticia social y el mal uso de los recursos por parte de las empresas y la sociedad.

Según Grimaldo (2018), la Responsabilidad Social (RS) puede definirse como el compromiso que tienen todos los ciudadanos, las instituciones - 
públicas y privadas - y las organizaciones sociales, para contribuir al aumento del bienestar de la sociedad.

Figura 1: Detalle del desarrollo de la metodología
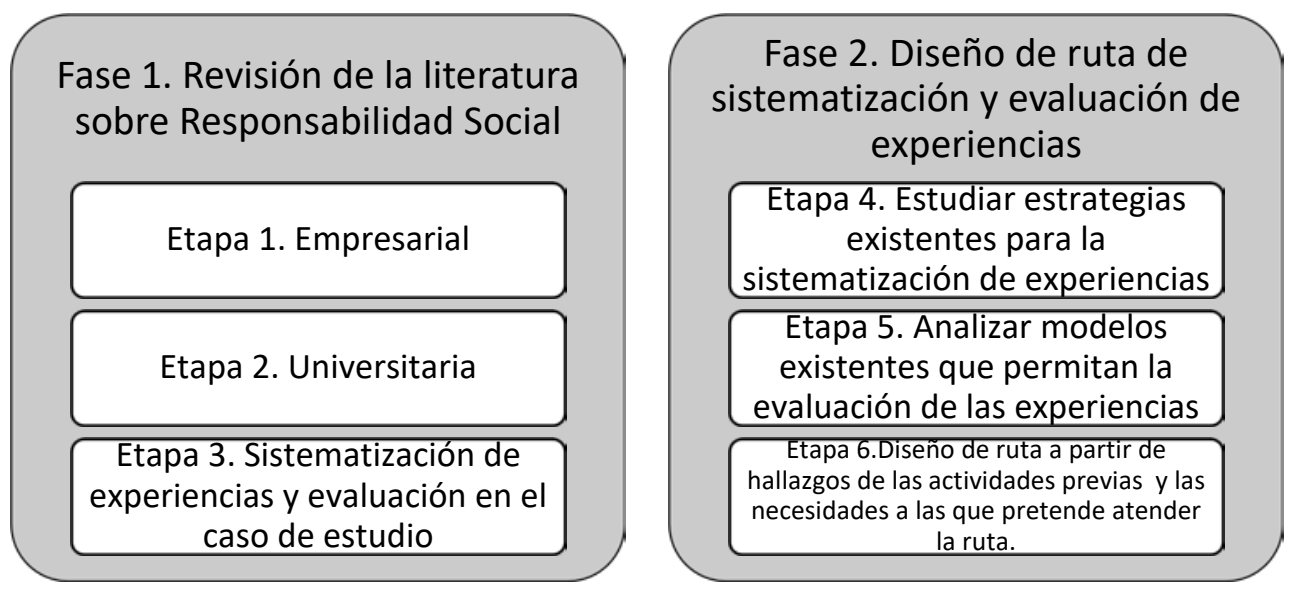

Fuente: Elaboración propia

De acuerdo con Server y Villalonga (2005), el término Responsabilidad Social se origina en Estados Unidos a finales de la década de los cincuenta y principios de los sesenta, a partir del reconocimiento del hecho de que las empresas, "además de producir bienes y servicios, eran responsables de los riesgos sanitarios de sus trabajadores y de la contaminación que generaban". Los informes en donde se documentaba la información relativa a los impactos externos a la empresa, dieron origen a lo que hoy se conoce como "balances sociales", cuyo uso se difundió de Estados Unidos a Europa.

En los últimos años el concepto ha evolucionado de la búsqueda de beneficios puramente económicos a aquellos de nivel medioambiental y social.

\section{Responsabilidad Social Empresarial}

La Responsabilidad Social Empresarial (RSE) se conformó como concepto a partir de determinadas exigencias sociales y del mercado (Almeida-Pástor y Arrechavaleta-Guarton, 2018). Uno de sus primeros exponentes, Friedman (1962), la definió con base en la responsabilidad de las empresas con sus accionistas. 
Jones y Rupp (2018) mencionan que la RSE no tiene una única definición ampliamente aceptada. De acuerdo con Rodríguez et al. (2015), la dificultad para lograr una definición concreta es lo dinámico del término, que cambia con el tiempo, de acuerdo con el devenir de las empresas y los retos a los que se ven enfrentadas según su entorno.

La Comisión Europea en el Libro Verde (2001) define la RSE como "la integración voluntaria, por parte de las empresas, de las preocupaciones sociales y ambientales en sus operaciones empresariales y en sus relaciones con sus interlocutores". Para Jácome-Lara et al. (2018), la RSE involucra un compromiso que incluye elementos legales y éticos derivados de los impactos generados a nivel social, medioambiental, laboral como consecuencia de la actividad organizacional. En palabras de BermúdezColina y Mejías-Acosta (2018) es un modelo cuyo elemento transversal en la gestión es el comportamiento responsable.

En las diversas aproximaciones desde campos como la sociología, economía y sicología entre otros, la RSE involucra ciertas características comunes entre las que se encuentran la existencia de políticas y prácticas organizacionales destinadas a beneficiar a las personas y otras entidades más allá de los dueños de negocios y accionistas, el desarrollo de acciones y comportamientos concretos por parte de las personas de la organización y la naturaleza discrecional de las actividades de RSE orientadas al bien social (Jones y Rupp, 2018).

La RSE implica la evaluación del impacto económico, social y ambiental de la empresa, tomando medidas para mejorarla de acuerdo con los requisitos de las partes interesadas e informando sobre mediciones relevantes. Este proceso de evaluación, realizado de forma continua, permitirá tomar acciones concretas hacia el cumplimiento de los objetivos trazados.

\section{Responsabilidad Social Universitaria}

El término Responsabilidad Social Universitaria (RSU) se originó, además de las necesidades de conocimiento de la empresa, como respuesta al desarrollo social de las localidades, países o regiones (Almeida y Arrechavaleta, 2018)

El documento de la Conferencia Mundial de Educación Superior realizada por la UNESCO en 1998, menciona que "los establecimientos de enseñanza superior, el personal y los estudiantes universitarios deberán: [...] aportar su 
contribución a la definición y tratamiento de los problemas que afectan al bienestar de las comunidades, las naciones y la sociedad mundial" UNESCO (1998). En la Conferencia Mundial de Educación Superior realizada en París en el año 2009, nuevamente, se afirma que la educación superior tiene la responsabilidad social de incrementar el entendimiento en distintas dimensiones, como son lo social, económico, científico y cultural, así como la capacidad de respuesta frente a ellas (USTA, 2015).

La RSU propone que, como ente transformador de la sociedad, la universidad debe participar de forma activa, especialmente en los ámbitos de mayor vulnerabilidad social y económica (De la Cruz y Sasía, 2008). Es por eso que la academia, mediante sus funciones sustantivas, debe motivar a la reflexión y acción a favor del desarrollo de la comunidad y el entorno del que hace parte.

Entre los casos indagados sobre RSU, se incluyen el proyecto Universidad Construye País, de Chile, desde donde se originó una definición de RSU; la generación de una Red Universitaria en RSU en la ciudad de Santiago de Cali; los proyectos de Universidad de Antioquia y Universidad Cooperativa de Colombia; el desarrollo del Modelo Estructural y Dinámico de Responsabilidad Social, Ética y Desarrollo, en la Universidad de Ibagué-Coruniversitaria y la construcción del êthos universitario de la Universidad Industrial de Santander como principio de autorregulación para la definición y apropiación de su protocolo ético. Estas iniciativas surgieron a partir de una convocatoria realizada por el BID (Hernández y Saldarriaga, 2009)

Otras iniciativas incluyen la del Observatorio Iberoamericano de Responsabilidad Social Universitaria en las Instituciones Universitarias de Educación a Distancia (OIRSUD) que involucra universidades de España, Portugal y América Latina; la Asociación de Educación Superior para la Sostenibilidad (Europa); la Asociación de Educación Superior de Nueva Jersey para la Sostenibilidad (Estados Unidos); la Asociación Global de Educación Superior para la Sostenibilidad, que congrega asociaciones y forma un conglomerado de más de mil universidades y la Red Global de Innovación Universitaria originada por la UNESCO (FUNLAM, 2015)

Adicional a lo anterior, la creación del Observatorio de Responsabilidad Social Universitaria -ORSU-, en el cual se encuentran vinculadas diecisiete (17) universidades colombianas en el 2007; la consolidación de la Responsabilidad Social en la Universidad Externado de Colombia como 
parte la misión institucional en el 2005 y el desarrollo de múltiples proyectos alrededor de la RSU que muestran la relevancia que ha adquirido la perspectiva de RSU en el ámbito universitario.

A nivel de otras universidades, se encontraron iniciativas de RSU en la Universidad de Rosario con el instituto SERES y el Sistema de Gestión Ambiental, la Universidad de la Sabana con el proyecto "Sabana Centro, Una provincia sostenible", la Universidad Javeriana con el programa Misión País Colombia y los proyectos Prosofi y Panamazónico y la Universidad El Bosque con la creación del programa "Pequeños científicos".

\section{Responsabilidad Social Universitaria en la Universidad Santo Tomás de Colombia}

La Universidad Santo Tomás, con más de 400 años de historia, ha desempeñado un papel interesante en la sociedad a partir de sus actividades de enseñanza desde sus inicios, buscando adicionalmente ejercer su influencia procurando la ayuda a comunidades vulnerables mediante actividades de desarrollo comunitario y formación. Estas actividades, desarrolladas con fundamento en la filosofía institucional enfocada en la "transformación de la realidad y la búsqueda del bien común" (USTA, 2019), se han venido transformando y fortaleciendo a través del tiempo en campos de acción que han dado origen a seis estrategias de RSU: desarrollo comunitario, relaciones interinstitucionales, emprendimiento, educación continua, asesorías y consultorías y egresados.

En el documento marco de Proyección Social de la Universidad Santo Tomás, se hace referencia a los primeros esbozos de lo que sería la RSU en la Universidad, que quedaron expresados en el Estatuto Orgánico de la institución en 1985 (USTA, 2015). De igual forma se presenta la evolución de la Proyección Social a lo largo del tiempo y en concordancia con las exigencias de la dinámica de la Educación Superior en Colombia, siendo así la Universidad partícipe activa de las propuestas y desarrollos en torno a la RSU.

En la Universidad, "la proyección social es la intención final de las otras dos funciones sustantivas (docencia e investigación). Debe entenderse, por una parte, la formación por la universidad de las nuevas generaciones con un compromiso social y, por otra, la intervención o impacto social que tiene la universidad en su entorno" (USTA, 2015). 


\section{Plumella Educatival}

Con base en el Acuerdo 29 de 2019, documento institucional que establece la Política de RSU, se concibe la misma como un "instrumento necesario para la proyección social y la extensión universitaria" cuyo objetivo es "estimular en la comunidad universitaria la participación en los procesos de transformación social, política, económica y ambiental de los territorios" (USTA, 2019). La RSU se expresa en las seis estrategias mencionadas, las cuales buscan responder a las necesidades del contexto sociodemográfico de la región, articulando la academia y el ejercicio investigativo, en procura del mejoramiento de la calidad de vida de las comunidades. Es así como desde el currículo se plantean problemas reales que conectan las necesidades sociales con los proyectos académicos, permitiendo tanto a estudiantes como docentes desarrollar en conjunto con la comunidad experiencias que generen aprendizajes para pensar, analizar y crear soluciones relevantes y pertinentes al contexto. La pertinencia y resultados de estas experiencias brindan a la comunidad tomasina la oportunidad de hacer visibles los alcances de las mismas, aspecto que se materializa en programas radiales y encuentros de RSU, así como reconocimientos institucionales como la Excelencia Tomasina, en donde, además de dar a conocer lo hecho, se motiva a los docentes a continuar creciendo en el alcance de sus iniciativas.

Uno de los objetivos de la Unidad de Proyección Social en la Universidad Santo Tomás, es "sistematizar y documentar las experiencias de los procesos vividos en los territorios que posibilitan apropiarse de las experiencias, comprenderlas teóricamente y orientarlas hacia nuevos rumbos en una perspectiva transformadora y sostenible" (USTA, 2019).

A partir de los referentes mencionados, la USTA ha visualizado una interesante oportunidad de trabajar en la recopilación y análisis de experiencias de RSU con base en su trayectoria de desarrollo de las mismas en diversos territorios de la geografía colombiana.

\section{Sistematización de experiencias}

Según (Martinic, 2010), el concepto de sistematización no tiene con un significado único, ya que son diversos los enfoques utilizados para el abordaje de las experiencias.

Bajo el enfoque de la educación popular, cuyas raíces se encuentran en el escenario latinoamericano, la sistematización de experiencias se puede 
definir como una forma de investigación basada en un proceso de reconstrucción y reflexión analítica que busca construir conocimiento a partir de experiencias, "en el cual la ciencia produce conocimiento y la práctica lo que hace es aplicarlo" (Mejía, 2008). Según Alfredo Ghiso, protagonista de esta modalidad de investigación, se entiende la sistematización como un "esfuerzo consciente de capturar los significados de la acción y sus efectos; como lecturas organizadas de las experiencias, como teorización y cuestionamiento contextualizado de la praxis social, para poder comunicar el conocimiento producido. En resumen, si bien la sistematización no es un concepto unívoco, aparece como un tipo de tarea reflexiva, que todos podíamos hacer y que al recuperar organizadamente la práctica permitirá volver a intervenir en ella y en la realidad con mayor eficacia y eficiencia". (Ghiso, p.4, 1998). Para Marco Raúl Mejía:

La sistematización de experiencias ha revalorizado los saberes de la práctica y constituye una propuesta investigativa para hacerlos emerger como saberes que no sólo dan cuenta de las prácticas, sino que las transforman y las convierten en procesos de empoderamiento de actores, organizaciones y grupos humanos, que se reconocen productores de saber desde y para la acción. Mejía (2008, p.14)

La sistematización es un proceso que supone el tránsito por diferentes momentos o ejes de análisis, aunque no siempre de forma lineal o secuencial. Estos momentos, aspectos o elementos a ser documentados y analizados durante el proceso de sistematización, constituyen variables que debieran ser observadas en la experiencia a partir de la combinación de diferentes instrumentos de recolección de datos (entrevistas a los responsables del proyecto y población beneficiaria, observación de la operación del proyecto en terreno, análisis de documentos, informes y otras fuentes de información).

En la literatura, el proceso de sistematización debe aportar a la generación de saberes a partir de las experiencias y creación de soluciones, a partir de la reflexión crítica frente a las mismas. De acuerdo con Martínez y Vásquez (2016) "la adquisición de una conciencia crítica es un aspecto esencial para que los excluidos perciban la necesidad de emprender acciones colectivas que transformen su vida". Una característica de las experiencias de RSU es el trabajo con minorías, o población en situación de exclusión. 


\section{La evaluación de experiencias}

De acuerdo con Tapella y Rodriguez-Bilella (2014), la mayoría de los enfoques de evaluación ponen énfasis en la medición de los resultados o impactos de un proyecto o programa, por lo que lo esencial de una evaluación es la valorización de las acciones emprendidas en la práctica, y comprende, por ejemplo, la valorización del cumplimiento de metas y objetivos.

En la investigación evaluativa, se han desarrollado dos métodos: el cuantitativo que concibe la realidad social como una estructura objetiva y externa al sujeto y su contexto, buscando medir y establecer las relaciones causales que pueden existir entre distintas variables para explicar los sucesos de la realidad. El cualitativo, que se asocia a la tradición interpretativa, y afirma que "la realidad social por excelencia son los sentidos subjetivos que orientan la acción de los sujetos, se busca comprender una realidad social que se construye intersubjetivamente y donde las relaciones son complejas y multivariadas." Martinic, S. (2010)

\section{Comparación entre sistematización y evaluación}

Es importante hacer claridad entre los dos conceptos. Para la evaluación, lo esencial es la valorización de las acciones emprendidas en una práctica, cumplimiento de metas y objetivos, la relación costobeneficio en el uso de los fondos, por ejemplo. Para la sistematización lo principal es comprender el desarrollo de la experiencia y aprender de ésta (Morgan y Quiroz, 1988).

Tabla 2. Comparación entre Sistematización y Evaluación.

\begin{tabular}{lll}
\hline $\begin{array}{l}\text { Objeto de } \\
\text { estudio }\end{array}$ & $\begin{array}{l}\text { Sistematización } \\
\text { con énfasis en los procesos } \\
\text { y las estrategias de } \\
\text { intervención. }\end{array}$ & $\begin{array}{l}\text { Un proyecto con la } \\
\text { comparación de los resultados } \\
\text { alcanzados frente a lo } \\
\text { planificado, de acuerdo a } \\
\text { criterios de eficiencia, eficacia, }\end{array}$ \\
& $\begin{array}{l}\text { Responde a ¿qué pasó? Y } \\
\text { ¿cómo se hizo? }\end{array}$ & $\begin{array}{l}\text { impacto, sostenibilidad. } \\
\text { Responde a ¿cuánto se ha } \\
\text { hecho? }\end{array}$ \\
\hline Propósitos & Recuperar, reconstruir, & $\begin{array}{l}\text { Valorar los resultados de una } \\
\text { experiencia en términos del }\end{array}$ \\
& organizar y procesar & cambio producido, de modo \\
& experiencias interpretando \\
críticamente los hechos, & que la información y el \\
para obtener de ellas & \\
\hline
\end{tabular}




\begin{tabular}{|c|c|c|}
\hline & $\begin{array}{l}\text { aprendizajes y lecciones que } \\
\text { permitan mejorar las } \\
\text { prácticas. }\end{array}$ & $\begin{array}{l}\text { aprendizaje resultante mejoren } \\
\text { la toma de decisiones. }\end{array}$ \\
\hline Métodos & $\begin{array}{l}\text { Requiere de métodos que } \\
\text { permiten pasar de la } \\
\text { experiencia vivida a la } \\
\text { comunicación y aprendizaje. } \\
\text { Utiliza procedimientos y } \\
\text { técnicas que se asocian a la } \\
\text { reconstrucción, reflexión y } \\
\text { análisis, así como de } \\
\text { interpretación crítica. }\end{array}$ & $\begin{array}{l}\text { Utiliza procedimientos y } \\
\text { técnicas que se asocian a } \\
\text { métodos cualitativos y } \\
\text { cuantitativos. Decide entre } \\
\text { unos u otros o combina ambas } \\
\text { metodologías, tomando en } \\
\text { cuenta la naturaleza de los } \\
\text { hechos a evaluar. }\end{array}$ \\
\hline
\end{tabular}

Fuente: Adaptado de Berdegué, Ocampo y Escobar (2007)

Martinic (2010) afirma que la sistematización contribuye "al desarrollo del referencial que necesita la evaluación para la interpretación de las prácticas y la evaluación apoyará a la sistematización en su comprensión y verificación de los resultados e impactos de las experiencias."

Se tomará como referente de comparación entre sistematización y evaluación lo propuesto por Berdegué, Ocampo y Escobar (2007), y sintetizado en la tabla 2.

\section{Estrategias para la Sistematización de las experiencias}

De acuerdo con Mejía (2012), la práctica "emerge como un lugar en el cual los saberes están en la acción, pero también como un lugar de creación de saberes". En este sentido, las experiencias de RSU se constituyen en elemento esencial para poner en práctica lo aprendido en el aula, pero más aún, en oportunidades para aprender, habiendo aplicado. De allí, los aprendizajes son readecuados, reorganizados, reformulados y transformados por la misma práctica.

Dada la importancia para la Universidad de fortalecer sus acciones en RSU, y brindar un marco de acción para los actores de la comunidad universitaria en pro de visibilizar y apoyar la mejora de forma sostenida de las experiencias realizadas, se realizó una revisión de algunas guías metodológicas de sistematización existentes, que permitieran contar con bases para proponer la ruta de sistematización y evaluación acorde a las necesidades de la universidad. 
En primer lugar, se revisó la propuesta de Barnechea y Morgan (2007), quienes, interesadas en brindar "orientaciones para desarrollar un proceso riguroso de producción de conocimientos y que sea asequible a desean emprender este esfuerzo", plantearon un método con base en su experiencia. Para estas autoras método no es una sucesión de procedimientos que deben ser aplicados mecánicamente, sino un conjunto de lineamientos que buscan orientar el razonamiento de los sistematizadores.

Así, Barnechea y Morgan (2007), proponen los siguientes pasos:

1. Diseño del plan de sistematización

2. Unificación de criterios con relación a la sistematización y lo que se espera lograr de ella: Producir conocimientos, ordenar la práctica, verificar la práctica, legitimar o validar una opción metodológica.

3. Definición de la experiencia a sistematizar

4. Reconstrucción de la experiencia

5. Análisis e interpretación de lo sucedido para comprenderlo

6. Comunicación de los resultados de la sistematización

Para las autoras, "la sistematización representa una articulación entre teoría y práctica, lo cual (...) pone ante el reto de desarrollar un lenguaje que exprese, a un nivel conceptual, toda la riqueza y la dinámica presentes en las prácticas de promoción" Barnechea y Morgan (2007).

Algunos elementos que sobresalen en su propuesta son la apuesta por la creatividad y la flexibilidad, en donde el método debe adaptarse a la experiencia y a las personas que están desarrollando el proceso. Se promueve la actitud crítica y reflexiva frente a la práctica y al método mismo.

La segunda propuesta analizada fue la de Jara (2011), quien hace énfasis en la obtención de aprendizajes críticos de las experiencias. De ahí el nombre "sistematización de experiencias". Jara define las experiencias como procesos históricos y sociales dinámicos, complejos y únicos, que deben ser analizados buscando su transformación.

Tabla 3. Propuesta de sistematización de Jara (2011).

\begin{tabular}{ll}
\hline \multicolumn{1}{c}{ Pasos } & \multicolumn{1}{c}{ Elementos } \\
\hline 1. El punto de partida & Haber participado en la experiencia \\
& Tener registros de las experiencias \\
\hline 2. Las preguntas iniciales & ¿Para qué queremos hacer esta \\
& sistematización? \\
& ¿Qué experiencia(s) queremos sistematizar? \\
\hline
\end{tabular}




\begin{tabular}{|c|c|}
\hline & $\begin{array}{l}\text { ¿Qué aspectos centrales de esas } \\
\text { experiencias nos interesa sistematizar? } \\
\text { ¿Qué fuentes de información vamos a } \\
\text { utilizar? } \\
\text { ¿Qué procedimientos vamos a seguir? }\end{array}$ \\
\hline $\begin{array}{l}\text { 3. Recuperación del proceso } \\
\text { vivido }\end{array}$ & $\begin{array}{l}\text { Reconstruir la historia } \\
\text { Ordenar y clasificar la información }\end{array}$ \\
\hline $\begin{array}{l}\text { 4. La reflexión de fondo: ¿por } \\
\text { qué pasó lo que pasó? }\end{array}$ & $\begin{array}{l}\text { Analizar y sintetizar. } \\
\text { Hacer una interpretación crítica del proceso }\end{array}$ \\
\hline 5. Los puntos de llegada & $\begin{array}{l}\text { Formular conclusiones } \\
\text { Comunicar los aprendizajes }\end{array}$ \\
\hline
\end{tabular}

Fuente: Elaboración propia, con base en Jara (2011).

Se identifican en Jara algunas recomendaciones como el haber participado de alguna en la experiencia que se va a sistematizar y llevar registros de lo que acontece durante la experiencia. Igual que en el caso anterior, la sencillez y flexibilidad en el proceso son características deseables para el desarrollo de la sistematización. Se destacan como apoyo las fichas para recopilar sucesos y aprendizajes, así como la matriz de ordenamiento y reconstrucción.

Es interesante en la propuesta evidenciar que la sistematización de experiencias se complementa con la evaluación y la investigación. La primera "normalmente se concentra en medir y valorar los resultados, aportando una interpretación crítica del proceso que posibilitó dichos resultados" Jara (2011) y la segunda "está abierta al conocimiento de muy diversas realidades y aspectos, aportando conocimiento vinculado a las propias experiencias particulares."

La tercera guía analizada fue la propuesta por Berdegué, Ocampo y Escobar (2007), orientada a la sistematización de experiencias locales de desarrollo rural. De acuerdo con estos autores la sistematización "Es un proceso de negociación de poder, por medio del cual grupos o sectores de la sociedad civil se involucran en dinámicas políticas para hacer valer sus intereses o sus formas de "hacer"” y "está dirigida a cambiar políticas (procedimientos, normas, formas de hacer, institucionalidad, etc.) en temas específicos a partir de propuestas puntuales".

Se plantean doce pasos en esta propuesta, que se listan a continuación:

1. Conformación del equipo de sistematización

2. Selección de la experiencia a sistematizar

3. Definición de los hitos de la experiencia 


\section{Plumilla Educatival}

4. Definición del eje de sistematización

5. Identificación de los actores involucrados en la experiencia

6. Recopilación, ordenamiento y análisis de la información y documentación disponibles

7. Formulación de preguntas e hipótesis de trabajo

8. Organización de entrevistas

9. Ordenamiento de la información recolectada

10. Taller grupal de retroalimentación

11. Redacción del informe de sistematización

12. Estrategia de comunicación y uso

En la aproximación realizada en esta tercera propuesta se destacan elementos como el aprovechamiento de la sistematización de experiencias con objetivos concretos de desarrollo de comunidades mediante el aprendizaje y la gestión del conocimiento. La sistematización proporciona información "para realizar un análisis crítico de los procesos de desarrollo impulsados por los proyectos" (Berdegué, Ocampo y Escobar, 2007). Las lecciones aprendidas propician modificaciones en las operaciones, métodos de trabajo o criterios normativos de los proyectos, que dan origen a mejoras en los proyectos subsiguientes.

\section{Revisión de modelos de evaluación de RSU}

Con el fin de contar con elementos de juicio que permitan evaluar la incidencia de las experiencias e RSU en los ámbitos en donde se realizan, así como visibilizar el impacto de las acciones proporcionando a las instituciones universitarias un medio de sistematización, se realizaron aproximaciones a modelos reconocidos y desarrollados por universidades en diferentes latitudes.

Los modelos utilizados se fundamentan en la revisión de Lima-Ravelo, Cabra-Copete, Tascón-Hoyos, (2018) y son: Modelo de Evaluación de la Innovación Social Universitaria Responsable (ISUR) desarrollada por la red Tuning América Latina, el modelo de la Asociación Universidades Confiadas a la Compañía de Jesús en América Latina (AUSJAL), el de la Pontifica Universidad Católica del Perú (PUCP) y el de Responsabilidad social universitaria: Manual de primeros pasos patrocinado por el BID. Un propósito común de los modelos de evaluación de RSU de acuerdo con Villa (2013), es contar con elementos de juicio para medir el impacto de las 
experiencias realizadas por la institución y fomentar el análisis y la reflexión conducente a implementar mejoras en la acción universitaria.

El modelo ISUR incluye un instrumento para evaluar los cambios experimentados internamente, y particularmente su impacto social. (Lima-Ravelo et al., 2018) y se identifican seis dimensiones o áreas de evaluación: curricular y pedagógica; organizacional; investigación, desarrollo e innovación; relación con el entorno; ambiental e internacionalización. (López, 2016)

El modelo AUSJAL construyó un sistema de autoevaluación apalancado en dos instrumentos: un sistema de indicadores institucionales y una encuesta de percepción de los diferentes actores afectados por la gestión universitaria. Estos instrumentos organizaron cinco dimensiones o áreas de impacto: educativo; cognoscitivo y epistemológico; social; organizacional y ambiental. (AUSJAL 2009)

El modelo de PUCP presenta un grupo de indicadores que cubren cuatro áreas: formación, investigación, gestión organizacional y vínculo con la sociedad. (Villa, 2013)

Finalmente, el modelo de Responsabilidad social universitaria: Manual de primeros pasos considera las dimensiones de impacto o ejes de: Campus responsable, formación profesional y ciudadana, gestión social del conocimiento y participación social (Vallaeys, de la Cruz y Sasía, 2009).

En la revisión de los modelos de RSU, se evidencia un trabajo mancomunado para construir un conjunto de factores que recojan las iniciativas y proyectos realizados, cuyo objetivo es aportar a la sociedad desde su quehacer. En general los modelos trabajan indicadores relacionados con la investigación, el currículo y la gestión organizacional entre otros y de manera particular es marcado el interés hacia la medición de impactos en la comunidad y el medio ambiente, temas alineados con los objetivos de desarrollo sostenible que hoy día hacen parte importante de las agendas de Responsabilidad Social en las empresas.

Como referencia para realizar la evaluación de experiencias de RSU se tuvo en cuenta el modelo propuesto por Lima-Ravelo, et al., (2018), en el cual se adaptan las seis dimensiones de RSU propuestas en el modelo ISUR (Innovación Social Universitaria Responsable), e incluye los factores de evaluación presentes en la Tabla 4. 
$-\infty$

Tabla 2. Modelo de evaluación de experiencias de RSU, Dimensiones y Factores.

\begin{tabular}{ll}
\hline \multicolumn{1}{c}{ Dimensión } & \multicolumn{1}{c}{ Factores evaluados } \\
\hline D1. Curricular y pedagógica & I. Formación del alumnado \\
& II. Desarrollo profesional para el personal \\
& universitario \\
& III. Respuesta educativa con equidad \\
& atendiendo a la \\
& diversidad \\
\hline D2. Organizacional & I. Relación con la sociedad, proyectos, \\
& contribución \\
& al desarrollo del país \\
& II. Trabajos en red \\
& III. Incidencia en políticas públicas \\
\hline D3. Investigación, desarrollo e & I. Políticas institucionales de investigación. \\
\hline innovación, I+D+i & I. Relación con la sociedad, proyectos, \\
\hline contribución \\
al desarrollo del país \\
II. Trabajos en red \\
III. Incidencia en políticas públicas \\
IV. Conocimiento académico popular \\
\hline I. Cultura ambiental. \\
III. Formación en el área del medio \\
ambiente. \\
V5. Acción en relación al medio ambiente \\
\hline D6. Internacionalización & I. Políticas de fomento de \\
internacionalización. \\
II. Trabajo de enfoque social en redes \\
internacionales. \\
III. Movilidad académica internacional. \\
IV. Programas de formación conjunta.
\end{tabular}

Fuente: Lima-Ravelo, et al. (2018)

\section{Resultados: Ruta para la sistematización y evaluación de experiencias de RSU}

Desarrollar la labor de sistematización y evaluación de las experiencias de RSU en la USTA constituye una valiosa fuente de información para visibilizar lo realizado, tomar decisiones que permitirán mejorar o profundizar en el desarrollo de las experiencias que generan transformación social en el entorno y contribuir de manera significativa al 
enriquecimiento de los currículos y la investigación aplicada a las regiones. Lo anterior reafirma nuestro objetivo, dado que según Cendales y Torres (2016), la sistematización "produce "teorías locales" sumamente pertinentes para las comunidades interpretativas en que se producen y claves para reorientar la acción."

Considerados los referentes bibliográficos estudiados tanto en la revisión de la literatura, como lo puntualmente desarrollado sobre estrategias sistematización y modelos de evaluación de experiencias, se propone una ruta para la sistematización y evaluación de experiencias de RSU con la que se busca además de lo inherente al proceso (de sistematizar y evaluar), mejorar, visibilizar y favorecer la réplica de estas actividades propias de quehacer universitario, esta ruta es sintetizada en la figura 2, en la que se resalta el hecho de que el proceso descrito es continuo.

\section{Figura 2. Ruta para la sistematización de experiencias en RSU.}

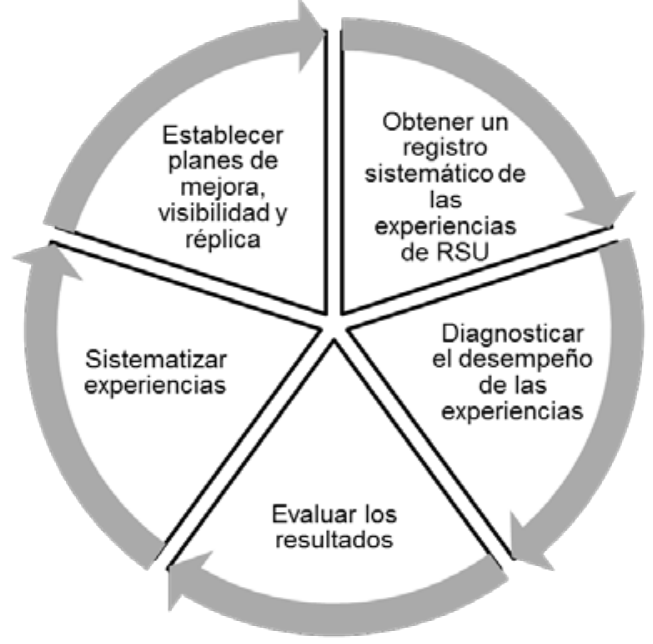

Fuente: Elaboración propia

En la figura 3 se presenta en detalle la ruta, mostrando no sólo las actividades, sino entradas y salidas de cada actividad, como parámetros de control del proceso. Aunque se asume una postura de trabajo cualitativa con base en la sistematización de experiencias, se introduce el uso de indicadores a partir del modelo de evaluación, que permitirá articular los trabajos de proyección social con proyectos de investigación, evaluación 
de actividad docente, entre otros elementos que hacen parte de la estrategia de Responsabilidad Social Universitaria y que requieren de resultados concretos y comparables.

Figura 3. Ruta para la sistematización de experiencias en RSU - detalle

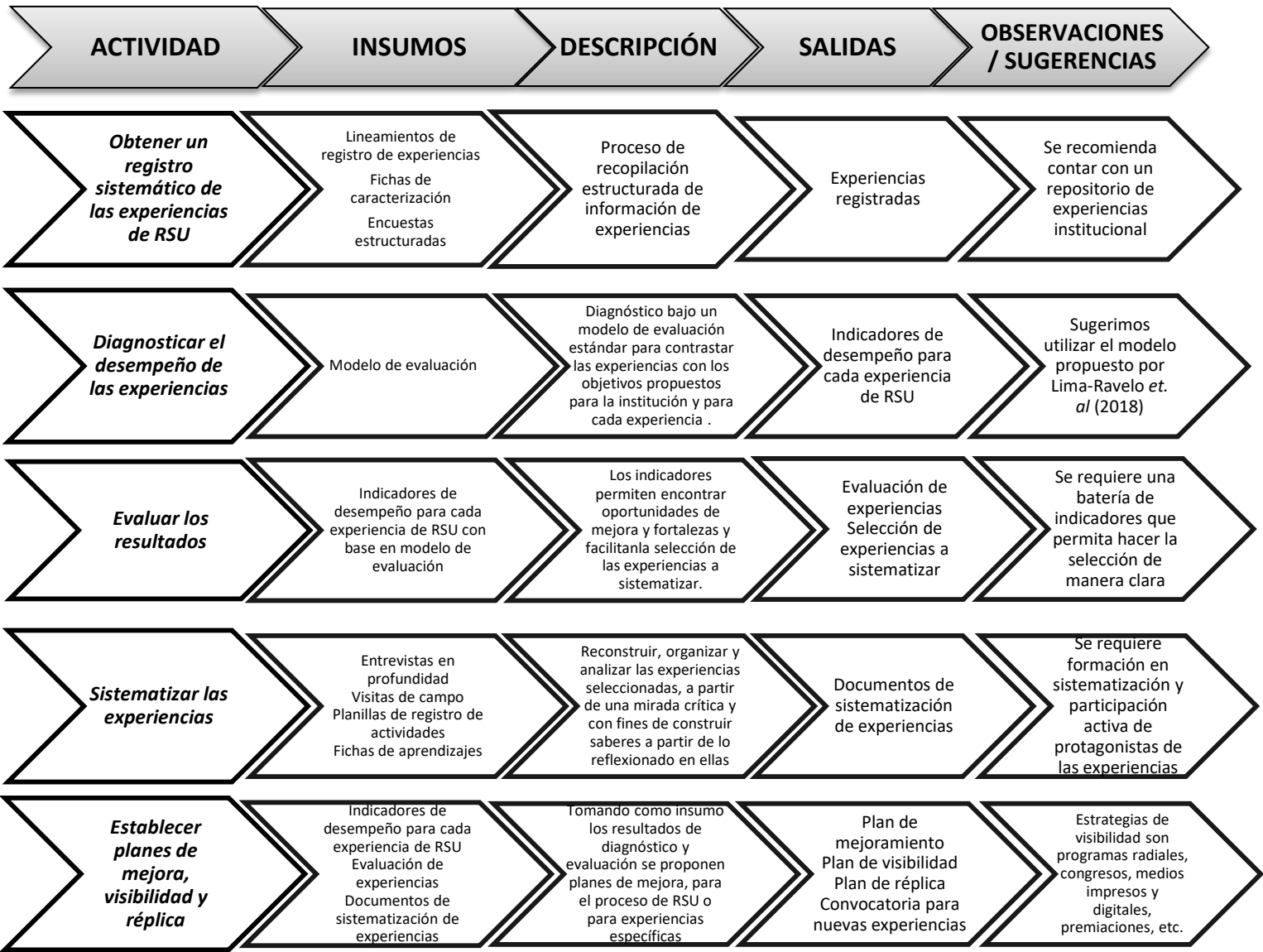

Fuente: Elaboración propia

Esta ruta se propone como un conjunto marco de lineamientos que orienta el proceso de sistematización y evaluación de experiencias de proyección social en la universidad, permitiendo contar con elementos para la recolección, recopilación, organización y análisis de la información y garantizando una base común para la comunidad académica y en especial al cuerpo docente y estudiantil que viven directamente las experiencias junto con las comunidades. 
Obtener un registro sistemático de las experiencias de RSU, permite la recopilación estructurada de la información de las experiencias. Para esto se ha de contar con lineamientos para el registro, fichas de caracterización y encuestas estructuradas dirigidas a los participantes de la experiencia. Como resultado de esta actividad se tendrá un número importante de experiencias registradas con lo que se recomienda la conformación de un repositorio institucional.

Diagnosticar el desempeño de las experiencias, se debe dar bajo un modelo de evaluación idóneo y estándar que permita contrastar las experiencias desarrolladas con los objetivos propuestos tanto para la institución como para cada experiencia en particular. Se sugiere utilizar el modelo propuesto por Lima-Ravelo et. al (2018). Resultado de esta actividad se obtendrán indicadores del desempeño de las experiencias.

La evaluación de resultados parte del análisis y reflexión los indicadores previamente generados para cada experiencia. Esto conduce a encontrar oportunidades de mejora y fortalezas y facilita la selección de aquellas experiencias que serán sistematizadas.

Sistematizar las experiencias consiste en reconstruir, organizar, analizar y documentar las experiencias seleccionadas, a partir de una mirada crítica y con fines de construir saberes a partir de lo reflexionado. Esto podrá hacerse con el apoyo de entrevistas en profundidad, visitas de campo, planillas de registro de actividades y fichas de aprendizajes; se requiere formación en sistematización y participación de los protagonistas de las experiencias. De gran relevancia en este proceso es la participación activa de los protagonistas de las experiencias, procesos educativos y de proyección social orientados y apoyados por la Universidad. Estos protagonistas, se convierten en generadores de conocimiento, que junto con la comunidad educativa pueden aportar de manera interesante al fortalecimiento de la estrategia y a mantener activos los proyectos, dado su interés más allá de lo académico. Esto brindará una base permanente de insumos para la siguiente etapa de la ruta.

Establecer planes de mejora, visibilidad y réplica. Tomando como insumo los resultados del diagnóstico y la evaluación se determinan planes de mejora, bien sea para el proceso institucional de RSU o para experiencias específicas; se eligen aquellas experiencias a ser visibilizadas y/o replicadas. Como resultado se obtiene el plan de mejoramiento de experiencias, así como lineamientos para la convocatoria de otras nuevas. 
Algunas estrategias para propiciar la visibilidad son los programas radiales, la participación en congresos, divulgación en medios impresos y digitales, premiaciones, entre otras.

Las acciones propuestas en esta ruta buscan favorecer el desarrollo y la transformación social a través de los esfuerzos que hacen las universidades en sus actividades de RSU, garantizando un desarrollo sistemático, ordenado y enfocado de las mismas. Estas actividades, que se realizan mediante la intervención pedagógica, el trabajo mancomunado entre docentes, estudiantes y sociedad y que propenden por un mejoramiento sostenido de las condiciones que se consideran adversas o limitantes, son grandes hitos que en la actualidad se visibilizan solamente como hechos y datos para los registros de documentos institucionales o de evaluación. Con la apropiación de la ruta y la definición de sus instrumentos, entre los cuales se encuentra el tablero e indicadores y el modelo de evaluación, se espera contar con más elementos para la interpretación crítica de las experiencias, comprensión de las realidades, recopilación de aprendizajes, mejora de los proyectos y potenciación de los cambios logrados, así como gestión de nuevo conocimiento para incorporarlo a nuevas experiencias y a los procesos de enseñanza-aprendizaje e investigación.

\section{Conclusiones}

El concepto y aplicación de Responsabilidad Social se ha fortalecido bastante en los últimos años en las universidades, lo cual ha hecho que las iniciativas y experiencias que se han venido trabajando con la comunidad requieran un marco de actuación que permita potencializarlas y hacerlas más evidentes. En el caso específico de la Universidad Santo Tomás, dados tanto el interés permanente por aportar de forma activa en la solución de problemas que afectan el bienestar de la comunidad como la amplia trayectoria en proyectos de intervención en comunidades expresada en las acciones de proyección social, la Responsabilidad Social se visibiliza como parte de su esencia, fuente de inspiración y centro de su propósito. Lo anterior, aunado la amplia cobertura de sus acciones a nivel nacional, evidencia la necesidad e importancia de registrar y visibilizar logros, dificultades y oportunidades que brinden trascendencia a la labor de proyección social que se realiza. 
La sistematización de las experiencias, desde su enfoque cualitativo, permite analizar situaciones que van más allá de los resultados finales de una experiencia o proyecto de proyección social. Es una fuente de aprendizajes, reflexión y apropiación de saberes sobre las prácticas y construcción de conocimiento que enriquece las funciones sustantivas. Brinda elementos de mejora para las experiencias, sus métodos y visibiliza sus alcances.

La sistematización y la evaluación proporcionan elementos complementarios que aportan información acerca de resultados, impactos, aprendizajes y concienciación acerca de las experiencias de RSU. La sistematización brinda el desarrollo conceptual y cualitativo, se convierte en insumo para la evaluación, en cuanto la evaluación aporta a la sistematización la posibilidad de valorar los resultados e impactos de las experiencias en las dimensiones que sean consideradas. Instrumentos como el modelo propuesto generan indicadores para identificar aciertos $u$ oportunidades de mejora que retroalimentan las experiencias.

La ruta de sistematización y evaluación puede constituir un punto de encuentro entre la investigación cuantitativa y cualitativa que permite abordar proyectos y experiencias de proyección social desde diferentes perspectivas y ayuda a lograr una visión holística de los problemas y sus soluciones. En ese sentido, provee elementos para desarrollar estrategias didácticas e investigativas que manejen las experiencias con enfoque transversal desde diferentes áreas del saber.

El proceso de desarrollo de una ruta para sistematizar y evaluar experiencias, proporcionó elementos desde diversas perspectivas enriqueciendo el modelo de medición construido en la primera fase del proyecto. La ruta propuesta se encamina a dar visibilidad las experiencias de RSU tanto al interior de la universidad como en la sociedad para favorecer su réplica y mejora. Los aprendizajes obtenidos y el registro sistemático de los mismos permitirán hacer aportes concretos y continuados en beneficio de las comunidades. 


\section{Plumilla Edueatiua}

\section{Referencias}

Almeida-Pástor, M. y Arrechavaleta-Guarton, N. (2018). Business Social Responsibility and its Restrictions in the University Academic Context. Revista Cubana de Educación Superior, 37, 87-103.

AUSJAL. (2014). Políticas y sistemas de autoevaluación y gestión de la Responsabilidad Social Universitaria en AUSJAL. Córdoba: Universidad Católica de Córdoba.

Barnechea, M., Gonzalez, E. y Morgan, M. (1992). ¿Y cómo lo hace? Propuesta de método de sistematización Taller Permanente de Sistematización. Lima: CEAAL.

Barnechea, M. y Morgan, M. (2007). "El conocimiento desde la práctica y una propuesta de método de sistematización de experiencias", Tesis de Maestría en sociología, Pontificia Universidad Católica Del Perú.

Berdegué, J. Ocampo, A. y Escobar, G. (2007). Guía Metodológica para la sistematización de experiencias locales de desarrollo rural. Lima: FIDAMERICA Y PREVAL.

Bermúdez-Colina, Y., y Mejías-Acosta, A. (2018). Medición de la responsabilidad social empresarial: casos en pequeñas empresas Latinoamericanas. Ingeniería Industrial, 39, 315-325.

Cendales, L., y Torres, A. (2006). La sistematización como experiencia Investigativa y formativa. Revista La Piragua, 23.

De la Cruz, C., \& Sasía, P. (2008). La responsabilidad de la universidad en el proyecto de construcción de una sociedad. Revista Educación Superior y Sociedad, 13, 17-52.

Ghiso, A. (1998). De la práctica singular al dialogo con lo plural. Medellín: Funlam.

Grimaldo, H. (2018). Responsabilidad-deber social. En Grimaldo, H. et al. El rol de la educación superior de cara a los desafíos sociales de América Latina y el Caribe. Córdoba: UNESCO-Universidad Nacional de Córdoba. 11-15

Jácome-Lara I., Salazar-Corrales A., Borja-Brazales, Y. (2017). La responsabilidad social empresarial en la gestión administrativa. Dominio de las ciencias, 3, 1147-1158. DOI: 10.23857/dc.v3i3.532

Jara Holliday, O. (2011) - Orientaciones teórico-prácticas para la sistematización de experiencias. Alforja. 
Jones, D. A., \& Rupp, D. E. (2017). Social responsibility IN and OF organizations: the psychology of corporate social responsibility among organizational members. En D. S. Ones, N. Anderson, C. Viswesvaran, \& H. K. Sinangil (Eds.), The SAGE Handbook of Industrial, Work, and Organizational Psychology V3. Thousand Oaks: SAGE Publications, Inc. DOI: $10.4135 / 9781473914964 . n 16$

Lima-Ravelo W., Cabra-Copete M., Tascón-Hoyos D. (2018). Medición del impacto de Responsabilidad Social Universitaria. Caso Universidad Santo Tomás, Colombia. Revista de Ciencias Humanas y Sociales. 34, 935-963.

López, A. 2016. Propuesta de modelo de evaluación de la Innovación Social Universitaria Responsable (ISUR). Revista Estudios Sobre Educación, 30, 71-93.

Martínez y Vásquez (2016). Comunicación para la inclusión social de minorías. La clase mágica, un modelo de intervención para el cambio social. Revista OBETS, 11, 188-209.

Martinic, S. (2010). Las relaciones entre la evaluación y la sistematización: apuntes para una discusión teórica sobre el objeto. Programa de Doctorado en Ciencias de la Educación. Santiago: Universidad Católica de Chile.

Mejía, M. R. (2008). La sistematización como proceso investigativo o la búsqueda de la episteme de las prácticas. Medellín: CINDE.

Rodríguez, A., Moyano, J., \& Jiménez, J. (2015). Estado actual de la investigación en Responsabilidad Social Corporativa a nivel organizativo: consensos y desafíos futuros. Revista de Economía Pública, Social y Cooperativa, 85, 127-164.

Server, R. y Villalonga, I. (2005). La Responsabilidad Social Corporativa (RSC) y su Gestión Integrada. Revista de Economía Pública, Social y Cooperativa, 53, 137-161.

UNESCO (1998). Declaración mundial sobre la educación superior en el siglo XXI: visión y acción. EDUCACIÓN SUPERIOR y SOCIEDAD. VOL. $9 \mathrm{~N}^{\circ} 2$ : 97-113

USTA - Universidad Santo Tomás. (2015). Documento marco de Proyección Social. Bogotá: Ediciones USTA.

USTA - Universidad Santo Tomás. (2019). Modelo Integral para la Acción. Bogotá: Universidad Santo Tomás. 
Vallaeys, F., De la Cruz, C., y Sasía, P. (2009). Responsabilidad social universitaria: Manual de primeros pasos. BID. México: McGraw Hill. Villa, A., Arnau, E., Cabezas, C., Cancino, R., Fernández-Lamarra, N. Greising, C., ... López, A. (2013). Un modelo de evaluación de Innovación Social Universitaria Responsable. Bilbao: Universidad de Deusto.

Recibido: 11 de junio de 2020

Aceptado: 27 de julio de 2021 